\title{
Effects of Creativity and Cluster Tightness on Short Text Clustering Performance
}

\author{
Catherine Finegan-Dollak ${ }^{1}$ \\ Xiangyi $\mathbf{Y e}^{2}$ \\ Reed Coke $^{1} \quad$ Rui Zhang ${ }^{1}$ \\ Dragomir Radev ${ }^{1}$ \\ \{cfdollak, reedcoke, ryanzh, yexy, radev\}@umich.edu \\ ${ }^{1}$ Department of EECS, University of Michigan, Ann Arbor, MI USA \\ ${ }^{2}$ Department of Mechanical Engineering, University of Michigan, Ann Arbor, MI USA
}

\begin{abstract}
Properties of corpora, such as the diversity of vocabulary and how tightly related texts cluster together, impact the best way to cluster short texts. We examine several such properties in a variety of corpora and track their effects on various combinations of similarity metrics and clustering algorithms. We show that semantic similarity metrics outperform traditional $n$-gram and dependency similarity metrics for kmeans clustering of a linguistically creative dataset, but do not help with less creative texts. Yet the choice of similarity metric interacts with the choice of clustering method. We find that graphbased clustering methods perform well on tightly clustered data but poorly on loosely clustered data. Semantic similarity metrics generate loosely clustered output even when applied to a tightly clustered dataset. Thus, the best performing clustering systems could not use semantic metrics.
\end{abstract}

\section{Introduction}

Corpora of collective discourse-texts generated by multiple authors in response to the same stimulus-have varying properties depending on the stimulus and goals of the authors. For instance, when multiple puzzle-composers write crossword puzzle clues for the same word, they will try to write creative, unique clues to make the puzzle interesting and challenging; clues for "star" could be "Paparazzi's target" or "Sky light." In contrast, people writing a descriptive caption for a photograph can adopt a less creative style. Corpora may also differ on how similar texts within a particular class are to one another, compared to how similar they are to texts from other classes. For example, entries in a cartoon captioning contest that all relate to the same cartoon may vary widely in subject, while crossword clues for the same word would likely be more tightly clustered.

This paper studies how such text properties affect the best method of clustering short texts. Choosing how to cluster texts involves two major decisions: choosing a similarity metric to determine which texts are alike, and choosing a clustering method to group those texts. We hypothesize that creativity may drive authors to express the same concept in a wide variety of ways, leading to data that can benefit from different similarity metrics than less creative texts. At the same time, we hypothesize that tightly clustered datasets-datasets where each text is much more similar to texts in its cluster than to texts from other clusters - can be clustered by powerful graph-based methods such as Markov Clustering (MCL) and Louvain, which may fail on more loosely clustered data. This paper explores the interaction of these effects.

Recently, distributional semantics has been popular and successful for measuring text similarity (Socher et al., 2011; Cheng and Kartsaklis, 2015; He et al., 2015; Kenter and de Rijke, 2015; Kusner et al., 2015; Ma et al., 2015; Tai et al., 2015; Wang et al., 2015). Word embeddings represent similar words in similar locations in vector space: "cat" is closer to "feline" than to "bird." It would be natural to expect such semantics-based approaches to be useful for clustering, particularly for corpora where authors have tried to express similar ideas in unique ways. And indeed, this paper will show that, depending on the choice of clustering method, semantics-based similarity 
measures such as summed word embeddings and deep neural networks can have an advantage over more traditional similarity metrics, such as n-gram counts, $\mathrm{n}$-gram tf-idf vectors, and dependency tree kernels, when applied to creative texts.

However, unlike in most text similarity tasks, in clustering the choice of similarity metric interacts with both the choice of clustering method and the properties of the text. Graph-based clustering techniques can be quite effective in clustering short texts (Rangrej et al., 2011), yet this paper will show that they are sensitive to how tightly clustered the data is. Moreover, the tightness of clusters in a dataset is a property of both the underlying data and the similarity metric. We show that when the underlying data can be clustered tightly enough to use powerful graph-based clustering methods, using semantics-based similarity metrics actually creates a disadvantage compared to methods that rely on the surface form of the text, because semantic metrics reduce tightness.

The remainder of this paper is organized as follows. Section 2 summarizes related work. Section 3 describes four datasets of short texts. In Section 4 , we describe the similarity metrics and clustering methods used in our experiments, as well as the evaluation measures. Section 5 shows that semantics-based similarity metrics have some advantage when clustering short texts from the most creative dataset, but ultimately do not perform the best when graph-based clustering is an option. In Section 6, we demonstrate the powerful effect that tightness of clusters has on the best combination of similarity metric and clustering method for a given dataset. Finally, Section 7 draws conclusions.

\section{Related Work}

The most similar work to the present paper is Shrestha et al. (2012), which acknowledged that the similarity metric and the clustering method could both contribute to clustering results. It compared four similarity methods and also tested four clustering methods. Unlike the present work, it did not consider distributional semantics-based similarity measures or similarity measures that incorporated deep learning. In addition, it reported that the characteristics of the corpora "overshadow[ed] the effect of the similarity measures," making it difficult to conclude that there were any significant differences between the similarity measures.

Several papers address the choice of similarity metric for short text clustering without varying the clustering method. Yan et al. (2012) proposed an alternative term weighting scheme to use in place of tf-idf when clustering using non-negative matrix factorization. King et al. (2013) used the cosine similarity between feature vectors that included context word and part-of-speech features and spelling features and applied Louvain clustering to the resulting graph. Xu et al. (2015) used a convolutional neural network to represent short texts and found that, when used with the k-means clustering algorithm, this deep semantic representation outperformed tf-idf, Laplacian eigenmaps, and average embeddings for clustering.

Other papers focused on choosing the best clustering method for short texts, but kept the similarity metric constant. Rangrej et al. (2011) compared k-means, singular value decomposition, and affinity propagation for tweets, finding affinity propagation the most effective, using tf-idf with cosine similarity or Jaccard for a similarity measure. Errecalde et al. (2010) describe an AntTreebased clustering method. They used the cosine similarity of tf-idf vectors as well. Yin (2013) also use the cosine similarity of tf-idf vectors for a twostage clustering algorithm for tweets.

One common strategy for short text clustering has been to take advantage of outside sources of knowledge (Banerjee et al., 2007; Wang et al., 2009a; Petersen and Poon, 2011; Rosa et al., 2011; Wang et al., 2014). The present work relies only on the texts themselves, not external information.

\section{Datasets}

Collective discourse (Qazvinian and Radev, 2011; King et al., 2013) involves multiple writers generating texts in response to the same stimulus. In a corpus of texts relating to several stimuli, it may be desirable to cluster according to which stimulus each text relates to-for instance, grouping all of the news headlines about the same event together. Here, we consider texts triggered by several types of stimuli: photographs that need descriptive captions, cartoons that need humorous captions, and crossword answers that need original clues. Each need shapes the properties of the texts.

Pascal and Flickr Captions. The Pascal Captions dataset (hereinafter PAS) and the $8 \mathrm{~K} \mathrm{Im}$ ageFlickr dataset (Rashtchian et al., 2010) are sets of captions solicited from Mechanical Turkers for photographs from Flickr and from the Pat- 
tern Analysis, Statistical Modeling, and Computational Learning (PASCAL) Visual Object Classes Challenge (Everingham et al., 2010).

PAS includes twenty categories of images (e.g., dogs, as in Example (1)) and 4998 captions. Each category has fifty images with approximately five captions for each image. We use the category as the gold standard cluster. The $8 \mathrm{~K}$ ImageFlickr set includes 38,390 captions for 7663 photographs; we treat the image a caption is associated with as the gold standard cluster. To keep dataset sizes comparable, we use a randomly selected subset of 5000 captions (998 clusters) from ImageFlickr (hereinafter FLK).

(1)

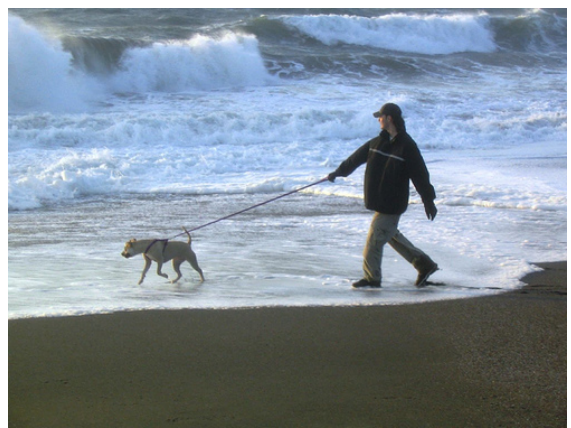

"a man walking a small dog on a very wavy beach" "A person in a large black coats walks a white dog on the beach through rough waves."

"Walking a dog on the edge of the ocean"

This task did not encourage creativity; instructions said to "describe the image in one complete but simple sentence." This could lead to sentences within a cluster being rather similar to each other. However, because photographs may contain overlapping elements-for instance, a photograph in the "bus" category of PAS might also show cars, while a photograph in the "cars" category could also contain a bus-texts in one cluster can also be quite similar to texts from other clusters. Thus, these datasets should not be very tightly clustered.

New Yorker Cartoon Captions. The New Yorker magazine has a weekly competition in which readers submit possible captions for a captionless cartoon (Example (2)) (Radev et al., 2015). We use the cartoon each caption is associated with as its gold standard cluster.

The complete dataset includes over 1.9 million captions for 366 cartoons. For this work, we use a total of 5000 captions from 20 randomly selected cartoons as the "TOON" dataset.

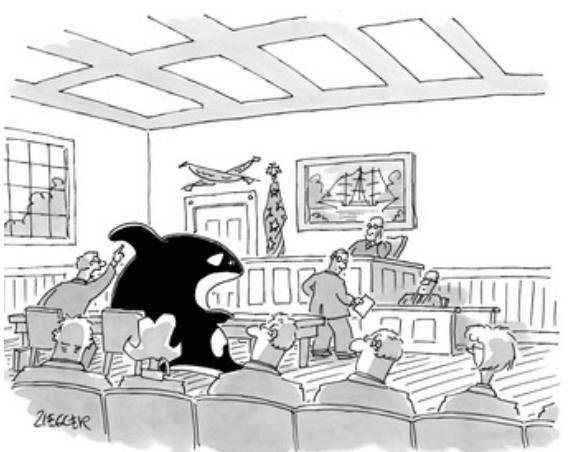

(2)

"Objection, Your Honor! Alleged killer whale."

"My client maintains that the penguin had a gun!"

"I demand a change of venue to a maritime court!"

Since caption writers seek to stand out from the crowd, we expect high creativity. This may encourage a more varied vocabulary than the FLK and PAS captions that merely describe the image. We also expect wide variation in the meanings of captions for the same cartoon, due to the different joke senses submitted for each, leading to low intra-cluster similarity. Moreover, some users may submit the same caption for more than one cartoon, so we can expect surprisingly high intercluster similarity despite the wide variation in cartoon prompt images. We therefore do not expect TOON to be tightly clustered.

Crossword Clues. A dataset of particularly creative texts is comprised of crossword clues. ${ }^{1}$ We use the clues as texts and the answer words as their gold standard cluster; all of the clues in Example (3) belong to the "toe" cluster.

\section{(3) Part of the foot \\ Little piggy \\ tic-tac- \\ The third $\mathrm{O}$ of $\mathrm{OOO}$}

The complete crossword clues dataset includes 1.7M different clues corresponding to 174,638 unique answers. The "CLUE" dataset includes 5000 clues corresponding to 20 unique answers selected by randomly choosing answers that have 250 or more unique clues, and then randomly choosing 250 of those clues for each answer.

Since words repeat, crossword authors must be creative to come up with clues that will not bore cruciverbalists. CLUE should thus contain many alternative phrasings for essentially the same idea. At the same time, there is likely to be relatively little overlap between clues for different answers, so CLUE should be tightly clustered.

\footnotetext{
${ }^{1}$ Collected from http: //crosswordgiant.com/
} 


\section{Method}

Here we describe the similarity metrics and clustering methods, as well as evaluation measures.

\subsection{Similarity Metrics}

We hypothesize that creative texts with wide vocabularies will benefit from similarity metrics based on semantic representation of the text, rather than its surface form. We therefore compare three metrics that rely on surface forms of words- $n$ gram count vectors, $\mathrm{tf}$-idf vectors, and dependency tree segment counts-to three semantic onessummed Word2Vec embeddings, LSTM autoencoders, and skip-thought vectors. In each case, we represent texts as vectors and find their cosine similarities; if cosine similarity can be negative, we add one and normalize by two to ensure similarity in the range $[0,1]$.

$N$-Gram Counts. First we consider $n$-gram count vectors. We use three variations: (1) unigrams, (2) unigrams and bigrams, and (3) unigrams, bigrams, and trigrams.

$N$-Gram tf-idf. We also consider weighting $n$-grams by tf-idf, as calculated by sklearn (Pedregosa et al., 2011).

Dependency Counts. Grammatical information has been found to be useful in text, particularly short text, similarity. (Liu and Gildea, 2005; Zhang et al., 2005; Wang et al., 2009b; Heilman and Smith, 2010; Tian et al., 2010; Šarić et al., 2012; Tai et al., 2015). To leverage this information, previous work has used dependency kernels (Tian et al., 2010), which measure similarity by the fraction of identical dependency parse segments between two sentences. Here, we accomplish the same effect using a count vector for each sentence, with the dependency parse segments as the vocabulary. We define the set of segments for a dependency parse to consist of, for each word, the word, its parent, and the dependency relation that connects them as shown in Example (4).

(4) Part of shoe

$$
\begin{array}{ll}
\text { a. } & \text { Segment 1: (part, ROOT, nsubj) } \\
\text { b. } & \text { Segment 2: (of, part, prep) } \\
\text { c. } & \text { Segment 3: (shoe, of, pobj) }
\end{array}
$$

Word2Vec. For each word, we obtain, if possible, a vector learned via Word2Vec (Mikolov et al., 2013) from the Google News corpus. ${ }^{2}$ We repre-

\footnotetext{
${ }^{2}$ https://code.google.com/archive/p/ word2vec/
}

sent a sentence as the normalized sum of its word vectors.

LSTM Autoencoder. We use Long ShortTerm Memory (LSTM) networks (Hochreiter and Schmidhuber, 1997) to build another semanticsbased sentence representation. We train an LSTM autoencoder consisting of an encoder network and a decoder network. The encoder reads the input sentence and produces a single vector as the hidden state at the last time step. The decoder takes this hidden state vector as input and attempts to reconstruct the original sentence. The LSTM autoencoder is trained to minimize the reconstruction loss. After training, we extract the hidden state at the last time step of encoder as the vector representation for a sentence. We use 300 -dimensional word2vec vectors pretrained on GoogleNews and generate 300-dimensional hidden vectors. LSTM autoencoders are separately trained for each dataset with $20 \%$ for validation.

Skip-thoughts (Kiros et al., 2015) trains encoder-decoder Recurrent Neural Networks (RNN) without supervision to predict the next and the previous sentences given the current sentence. The pretrained skip-thought model computes vectors as sentence representations.

\subsection{Clustering Methods}

We explore five clustering methods: k-means, spectral, affinity propagation, Louvain, and MCL.

K-means is a popular and straightforward clustering algorithm (Berkhin, 2006) that takes a parameter $k$, the number of clusters, and uses an expectation-maximization approach to find $k$ centroids in the data. In the expectation phase points are assigned to their nearest cluster centroid. In the maximization phase the centroids of are recomputed for each cluster of assigned points. Kmeans is not a graph-based clustering algorithm, but rather operates in a vector space.

Spectral clustering (Donath and Hoffman, 1973; Shi and Malik, 2000; Ng et al., 2001) is a graph-based clustering approach that finds the graph Laplacian of a similarity matrix, builds a matrix of the first $k$ eigenvectors of the Laplacian, and then applies further clustering to this matrix. The method can be viewed as an approximation of a normalized min-cuts algorithm or of a random walks approach. We use the default implementation provided by sklearn, which applies a Gaussian kernel to determine the graph Laplacian and uses 
$\mathrm{k}$-means for the subsequent clustering step.

Affinity propagation finds exemplars for each cluster and then assigns nodes to a cluster based on these exemplars (Frey and Dueck, 2007). This involves updating two matrices $R$ and $A$, respectively representing the responsibility and availability of each node. A high value for $R_{(i, k)}$ indicates that node $x_{i}$ would be a good exemplar for cluster $k$. A high value for $A_{(i, k)}$ indicates that node $x_{i}$ is likely to belong to cluster $k$. We use the default implementation provided by sklearn.

Louvain initializes each node to be its own cluster, then greedily maximizes modularity (Section 6.1 ) by iteratively merging clusters that are highly interconnected (Blondel et al., 2008).

Markov Cluster Algorithm (MCL) simulates flow on a network via random walk (Van Dongen, 2000). The sequence of nodes is represented via a Markov chain. By applying inflation to the transition matrix, the algorithm can maintain the cluster structure pronounced in the transition matrix of this random walk-a structure that would otherwise disappear over time. ${ }^{3}$

\subsection{Evaluation Methods}

Adjusted Rand Index We use the sklearn implementation of the Adjusted Rand Index (ARI) ${ }^{4}$ (Hubert and Arabie, 1985):

$$
\mathrm{ARI}=\frac{R I-\text { Expected } \mathrm{RI}}{\max R I-\text { Expected } \mathrm{RI}}
$$

where $R I$ is the Rand Index,

$$
R I=\frac{T P+T N}{T P+F P+F N+T N}
$$

$T P$ is the number of true positives, $T N$ is true negatives, and $F P$ and $F N$ are false positives and false negatives, respectively. The Rand Index ranges from 0 to 1 . ARI adjusts the Rand Index for chance, so that the score ranges from -1 to 1 . Random labeling will achieve an ARI score close to 0; perfect labeling achieves an ARI of 1 .

Purity is a score in the $[0,1]$ range that indicates to what extent sentences in the same predicted cluster actually belong to the same cluster. Given $\Omega=\left\{\omega_{1}, \omega_{2}, \ldots, \omega_{K}\right\}$, the predicted clusters, $C=\left\{c_{1}, c_{2}, \ldots, c_{J}\right\}$, the true clusters, and $N$, the number of examples, purity is

$$
\operatorname{Purity}(\Omega, C)=\frac{1}{N} \sum_{k \in K} \max _{j \in J}\left|\omega_{k} \cap c_{j}\right|
$$

\footnotetext{
${ }^{3}$ We use the implementation from http://micans. org $/ \mathrm{mcl} /$ with inflation=2.0.

${ }^{4}$ Equivalent to Cohen's Kappa (Warrens, 2008).
}

Normalized Mutual Information (NMI). We use the sklearn implementation of NMI:

$$
N M I(\Omega, C)=\frac{M I(\Omega, C)}{\sqrt{H(C) \cdot H(\Omega)}}
$$

The numerator is the mutual information (MI) of predicted cluster labels $\Omega$ and true cluster labels $C$. MI describes how much knowing what the predicted clusters are increases knowledge about what the actual classes are. Using marginal entropy $(H(x)$ ), NMI normalizes MI so that it ranges from 0 to 1. If $C$ and $\Omega$ are identical—that is, if the clusters are perfect-NMI will be 1 .

\section{Vocabulary Width}

\subsection{Descriptive Statistics for Vocabulary Width}

We predict that creative texts have a wider vocabulary than functional texts. We use two measures to reflect this wide vocabulary: the type/token ratio in the dataset (TTR), and that ratio normalized by the mean length of a text in the dataset.

TTR is an obvious estimate of the width of the vocabulary of a corpus. However, all other things being equal, a corpus of many very short texts triggered by the same stimulus would have more repeated words, proportional to the total number of tokens in the corpus, than would a corpus of a smaller number of longer texts. We might therefore normalize the ratio of types to tokens by dividing by the mean length of a text in the dataset, leading to the normalized type-to-token ratio (NTTR) and TTR values shown in Table 1.

\begin{tabular}{lcccc}
\hline & CLUE & TOON & PAS & FLK \\
\hline TTR & 0.1680 & 0.1064 & 0.0625 & 0.0561 \\
NTTR & 0.0377 & 0.0086 & 0.0058 & 0.0047
\end{tabular}

Table 1: Vocabulary properties of each dataset

FLK, PAS, and CLUE conform to expectations. The creative CLUE has TTR more than double that of the more functional PAS and FLK. The effect is more pronounced using NTTR. Surprisingly, TOON falls closer to the PAS and FLK end of the spectrum, suggesting that vocabulary width does not capture the creativity in the captioning competition; perhaps the creativity of cartoon captions is about expressing different ideas, rather than finding unique ways to express the same idea. For the experiments based on vocabulary width, we therefore compare PAS and CLUE. 


\subsection{Experiments}

We hypothesize that if a dataset uses a wide variety of words to express the same ideas, similarity metrics that rely on the surface form of the sentence will be at a disadvantage compared to similarity metrics based in distributional semantics. Thus, word2vec, LSTM autoencoders, and skip-thoughts ought to perform better than the $n$-gram-based methods and dependency count method when applied to CLUE, but should enjoy no advantage when applied to PAS.

We begin by comparing the performance of all similarity metrics on PAS and CLUE, using kmeans for clustering. We then also examine their performance with MCL.

\subsection{Results and Discussion}

Table 2 compares the performance of all similarity metrics on PAS and CLUE using k-means and MCL. Using k-means on PAS, the unigram tf-idf similarity metric gives the strongest performance for purity and NMI and came in a close second for ARI. LSTM slightly outperformed the other similarity metrics on ARI, but had middle-of-theroad results on the other evaluations. Overall, the semantics-based similarity metrics gave reasonable but not exceptional ARI and purity results, but were at the low end on NMI. This is consistent with our hypothesis that when authors are not trying to express creativity by using a wider vocabulary, surface-based similarity metrics suffice.

For k-means on CLUE, the picture is quite different: the semantics-based similarity metrics markedly outperformed any other similarity metric on ARI. LSTM also provides the best purity score, followed by skip-thought. The semantics-based metrics do not stand out for NMI, though. Based on these results, we conclude that semantics-based measures provide a significant advantage over traditional similarity metrics when using k-means on the wide-vocabulary, creative CLUE.

When clustering with MCL, however, the semantics-based methods perform exceptionally poorly on both datasets. Interestingly, the $n$-grambased similarity metrics performed very well when paired with MCL on CLUE-outperforming the best of the k-means scores-while the same metrics performed terribly with MCL on PAS.

We hypothesize that the semantics-based similarity metrics produce less tightly clustered data than the surface-form-based metrics do, and that this may make clustering difficult for some graphbased clustering methods. The next section describes how we test this hypothesis.

\section{Tightness of Clusters}

\subsection{Descriptive Statistics for Tightness}

Two pieces contribute to cluster tightness: the dataset itself and the choice of similarity metric. To illustrate, we represent each text with the vector for its similarity metric - for instance, the sum of its word $2 \mathrm{vec}$ vectors or the unigram tf-idf vectorand reduce it to two dimensions using linear discriminant analysis. We plot five randomly selected gold standard clusters. Plots for unigram tf-idf and word2vec representations of PAS and CLUE are shown in Figures 1 and 2. These support the intuition that semantics-based similarity metrics are not as tightly clustered as $n$-gram-based metrics. Note also that the CLUE unigram tf-idf clusters appear tighter than the PAS unigram tf-idf clusters.

To quantify this, we compute modularity (Newman, 2004; Newman, 2006): ${ }^{5}$

$$
Q=\frac{1}{2 m} \sum_{i j}\left(A_{i j}-\frac{k_{i} k_{j}}{2 m}\right) \delta\left(c_{i}, c_{j}\right)
$$

$A_{i j}$ is the edge weight between nodes $i$ and $j$. $\delta\left(c_{i}, c_{j}\right)$ indicates whether $i$ and $j$ belong to the same cluster. $m$ is the number of edges. $k_{i}$ is the degree of vertex $i$, so $\frac{k_{i} k_{j}}{2 m}$ is the expected number of edges between $i$ and $j$ in a random graph. Thus, modularity is highest when nodes in a cluster are highly interconnected, but sparsely connected to nodes in different clusters. We use this statistic in an unconventional way, determining the modularity of the golden clusters.

Table 3 shows the modularities for all four datasets using the unigram, trigram, unigram tfidf, trigram tf-idf, dependency, word2vec, and skipthoughts similarity metrics. As suggested by Figures 1 and 2, the CLUE surface-formbased similarities have the highest modularity by far. The surface-form-based similarities for all datasets have much higher modularity than any of the semantics-based similarities; indeed, the

\footnotetext{
${ }^{5}$ Newman (2010) notes that modularity for even a perfectly mixed network generally cannot be 1 and describes a normalized modularity formula. We calculated both normalized and non-normalized modularity and found the pattern of results to be the same, so we report only modularity.
} 
MCL

\begin{tabular}{|l|rrr|rrr|rrr|rrr|}
\hline \multicolumn{1}{c}{ PAS } & \multicolumn{3}{c}{ CLUE } & \multicolumn{4}{c}{ PAS } & CLUE \\
\hline Metric & ARI & Purity & NMI & ARI & Purity & NMI & ARI & Purity & NMI & ARI & Purity & NMI \\
\hline Unigram & 0.0286 & 0.141 & 0.110 & 0.0137 & 0.173 & 0.153 & $1.00 E-05$ & 0.058 & 0.051 & 0.0620 & 0.527 & 0.439 \\
Bigram & 0.0230 & 0.143 & 0.111 & 0.0124 & 0.165 & 0.142 & $2.50 E-05$ & 0.065 & 0.070 & 0.0835 & 0.585 & 0.465 \\
Trigram & 0.0289 & 0.139 & 0.108 & 0.0148 & 0.178 & 0.156 & $3.60 E-05$ & 0.069 & 0.081 & 0.1034 & 0.608 & 0.478 \\
Uni. tf-idf & 0.0445 & $\mathbf{0 . 1 8 9}$ & $\mathbf{0 . 1 6 9}$ & 0.0180 & 0.202 & 0.188 & $2.20 \mathrm{E}-05$ & 0.061 & 0.060 & $\mathbf{0 . 1 4 8 2}$ & 0.643 & 0.506 \\
Bi. tf-idf & 0.0287 & 0.158 & 0.135 & 0.0156 & 0.205 & 0.205 & $3.86 E-04$ & 0.104 & 0.135 & 0.1327 & 0.722 & 0.544 \\
Tri. tf-idf & 0.0345 & 0.176 & 0.142 & 0.0134 & 0.195 & $\mathbf{0 . 2 1 3}$ & $6.49 E-03$ & 0.212 & 0.230 & 0.1280 & $\mathbf{0 . 7 5 1}$ & $\mathbf{0 . 5 6 1}$ \\
Dependency & 0.0122 & 0.131 & 0.104 & 0.0071 & 0.169 & 0.207 & $\mathbf{2 . 0 7 E - 0 2}$ & $\mathbf{0 . 2 8 0}$ & $\mathbf{0 . 2 6 4}$ & 0.0832 & 0.745 & 0.543 \\
Word2Vec & 0.0274 & 0.142 & 0.103 & 0.0527 & 0.189 & 0.165 & 0.000 & 0.050 & 0.000 & 0.0000 & 0.050 & 0.000 \\
LSTM & $\mathbf{0 . 0 4 5 3}$ & 0.170 & 0.142 & $\mathbf{0 . 0 8 3 7}$ & $\mathbf{0 . 2 4 0}$ & 0.202 & 0.000 & 0.050 & 0.000 & 0.0000 & 0.050 & 0.000 \\
Skipthought & 0.0311 & 0.140 & 0.106 & 0.0691 & 0.215 & 0.180 & 0.000 & 0.050 & 0.000 & 0.0000 & 0.050 & 0.0009 \\
\hline
\end{tabular}

Table 2: A comparison of all similarity metrics on PAS and CLUE datasets, clustered using k-means and MCL. For all evaluations, higher scores are better.
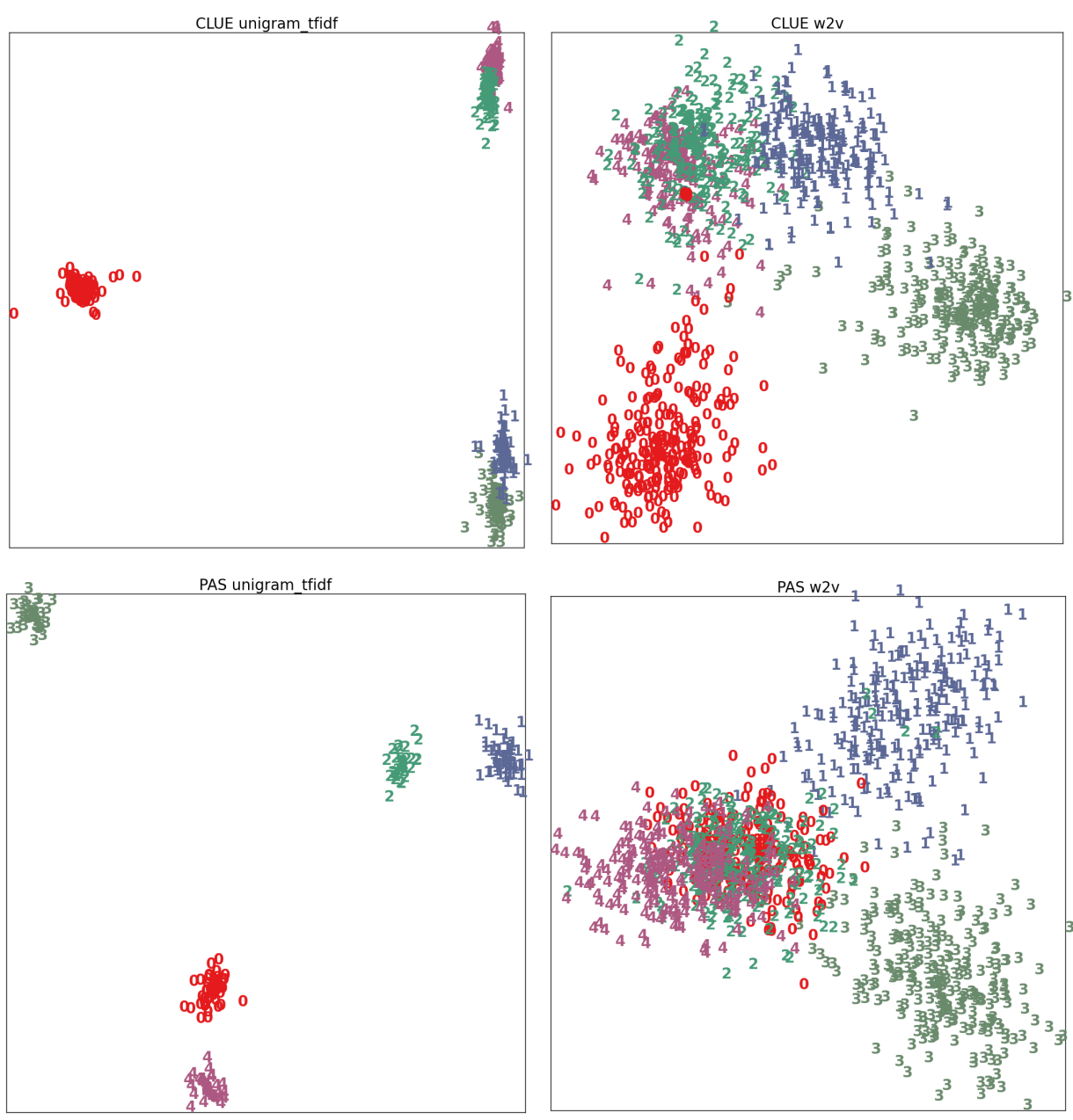

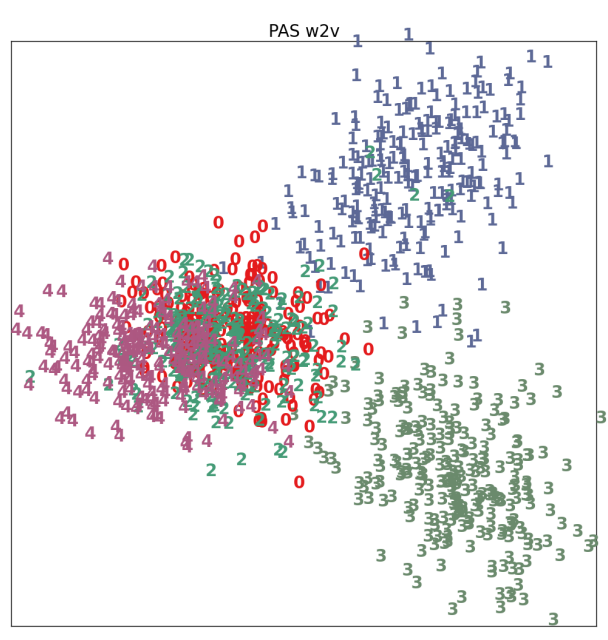

Figure 1: Plots of unigram tf-idf (left) and word2vec (right) vectors representing five randomly selected clusters of CLUE: clues for words "ets," "stay," "yes," "easel," and "aha."

Figure 2: Plots of unigram tf-idf (left) and word2vec (right) vectors representing five randomly selected clusters of PAS: images containing "bus," "boat," "car," "bird," and "motorbike." semantics-based similarities rarely have modularity much higher than zero. Thus, we conclude both that CLUE is more tightly clustered than the other datasets and that surface-form-based measures yield tighter clusters than semantics-based measures.

CLUE's tight clustering could be due in part to its particularly short texts. Additionally, it might reflect the semantics of the dataset: words that the clues hint at may be less similar to one another than the categories in PAS are to each other. For instance, some images in PAS's "bus" category include cars, and vice-versa.

The difference between semantics-based and surface-form similarity metrics likely arises from the fact that similarity of a word pair is a yes-or- 


\begin{tabular}{l|rrrr} 
Metric & PAS & Clues & TOON & FLK \\
\hline Unigram & 0.0254 & 0.1849 & 0.0214 & 0.0065 \\
Bigram & 0.0312 & 0.2216 & 0.0293 & 0.0103 \\
Trigram & 0.0347 & 0.2447 & 0.0352 & 0.0135 \\
Uni. tf-idf & 0.0587 & 0.3005 & 0.0519 & 0.0184 \\
Bi. tf-idf & $\mathbf{0 . 0 8 7 7}$ & 0.3875 & 0.0950 & 0.0394 \\
Tri. tf-idf & 0.0347 & 0.4339 & $\mathbf{0 . 1 3 1 1}$ & $\mathbf{0 . 0 6 1 8}$ \\
Dependency & 0.0799 & $\mathbf{0 . 4 7 2 9}$ & 0.0451 & 0.0299 \\
Word2Vec & 0.0020 & 0.0036 & 0.0008 & 0.0004 \\
LSTM & 0.0072 & 0.0121 & 0.0020 & 0.0009 \\
Skipthought & 0.0009 & 0.0028 & 0.0006 & 0.0003
\end{tabular}

Table 3: Modularity for all datasets

no question to surface-form-based metrics, but a question of degree to semantics-based ones. According to semantics-based methods, "cat" is more similar to "feline" than it is to "dog," but more similar to "dog" than to "motorcycle." This creates some similarity between texts from different clusters, blurring the lines between them. Thus, "The man walks his dog" and "A woman with a cat" are entirely dissimilar according to surface form methods, but not according to the semantics-based measures. Even if the nodes in a cluster are highly interconnected, if the connections between nodes in different clusters are too strong, modularity will be low.

To determine whether cluster tightness influences the best clustering method, we tested all clustering methods on all four datasets using unigram, trigram, unigram tf-idf, trigram tf-idf, word2vec, and skipthought similarity metrics.

\subsection{Results and Discussion}

As can be seen in Figure 3, the best ARI results by a large margin were those on the tightly clustered CLUE. Louvain, which provides the best ARI for CLUE, and MCL, which provides the second best, both performed most strongly when paired with the surface-form-based similarity metrics ( $n$-gram counts, tf-idf, and dependency count), which had high modularity relative to the semantics-based metrics. Although CLUE also differs from the other datasets in that it has the shortest mean text length, text length by itself cannot explain the observed differences in performance, since the pattern of graph-based clustering methods working best with modular data is consistent within each dataset as well as between datasets.

CLUE is also the only dataset where the semantics-based similarity metrics performed exceptionally well with any of the clustering methods. Recall from Table 1 that CLUE had a markedly wider vocabulary than any other dataset. This further supports our findings in Section 5.3 regarding how creativity affects the usefulness of semantics-based similarity metrics.

FLK, which had the lowest modularity, cannot be clustered by the spectral, Louvain, or MCL algorithms. K-means provides the strongest performance, followed by affinity propagation.

TOON has the worst ARI results. Its bestperforming clustering methods are the graphbased Louvain and MCL methods. Both perform well only when paired with the most modular similarity metrics. Louvain seems less sensitive to modularity than MCL does. MCL's best performance by far for TOON is when it is paired with trigram tf-idf, which also had the highest modularity; its performance when paired with the lowermodularity similarity metrics rapidly falls away. In contrast, Louvain fares reasonably well with the lower $n$-gram tf-idfs, which also had lower modularity than trigram tf-idf.

Louvain and MCL follow a similar pattern on PAS: both perform at their peak on the most modular similarity metric (dependency), but Louvain handles slightly less modular similarity metrics nearly as well as the most modular one, while MCL quickly falters.

K-means' performance is not correlated with modularity. This makes sense, as k-means is the only non-graph-based method. Methods like MCL, which is based on a random walk, may be stymied by too many highly-weighted paths between clusters; the random walk can too easily reach a different neighborhood from where it started. But k-means relies on how close texts are to centroids, not to other texts, and so would be less affected.

The fact that k-means nevertheless performs poorly on TOON suggests that this dataset may be particularly difficult to cluster. An interesting test would be to measure inter-annotator agreement on TOON.

\section{Conclusions and Future Work}

This work has shown that creativity can influence the best way to cluster text. When using k-means to cluster a dataset where authors tried to be creative, similarity metrics utilizing distri- 

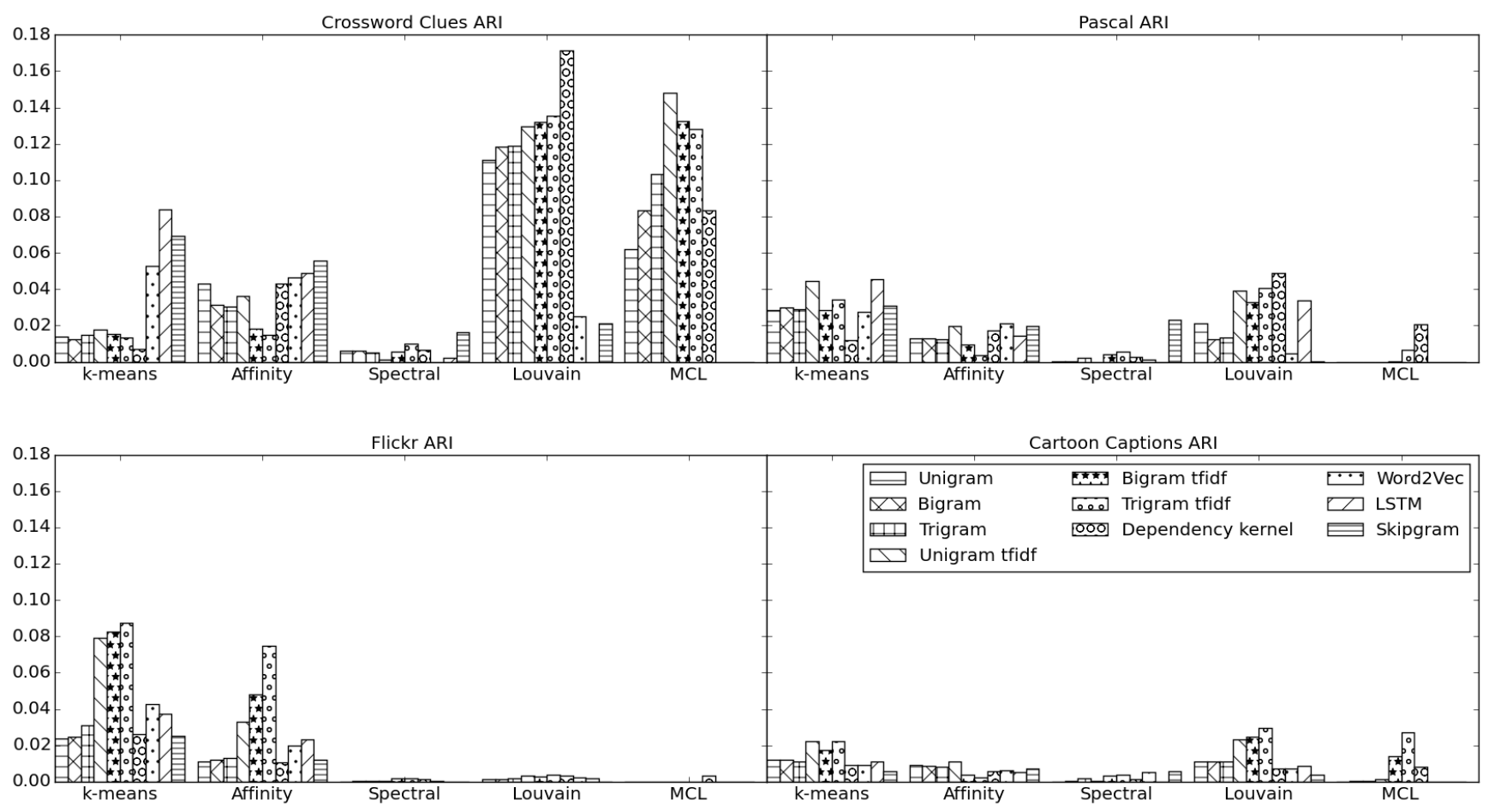

Figure 3: All similarity metrics and all clustering methods for the four datasets.

butional semantics outperformed those that relied on surface forms. We also showed that semanticsbased methods do not provide a notable advantage when applying k-means to less creative datasets. Since traditional similarity metrics are often faster to calculate, use of slower semantics-based methods should be limited to creative datasets.

Unlike most work on clustering short texts, we examined how the similarity metric interacts with the clustering method. Even for a creative dataset, if the underlying data is tightly clustered, the use of semantics-based similarity measures can actually hurt performance. Traditional metrics applied to such tightly clustered data generate more modular output that enables the use of sophisticated, graph-based clustering methods such as MCL and Louvain. When either the underlying data or the similarity metrics applied to it produce loose clusters with low modularity, the sophisticated graph clustering algorithms fail, and we must fall back on simpler methods.

Future work can manipulate datasets' text properties to confirm that a specific property is the cause of observed differences in clustering. Such work should alter the datasets TTR and NTTR while holding mean length of texts constant. A pilot effort to use word embeddings to alter the variety of vocabulary in a dataset has so far not succeeded, but future experiments altering vocabulary width or modularity of a dataset and finding that the modified dataset behaved like natural datasets with the same properties could increase confidence in causality. Future work can also explore finer clusters within these datasets, such as clustering CLUE by word sense of the answers and TOON by joke sense.

These results are a first step towards determining the best way to cluster a new dataset based on properties of the text. Future work will explore further how the goals of short text authors translate into measurable properties of the texts they write, and how measuring those properties can help predict which similarity metrics and clustering methods will combine to provide the best performance.

\section{Acknowledgments}

The authors are grateful for the help and insights of Jonathan Kummerfeld, Spruce Bondera, Kyle Bouwens, Kurt McEwan, Francisco Rivera Reyes, Clayton Thorrez, Chongruo Wu, Yue Xu, Harry Zhang, and Joel Tetreault. We appreciate the comments of the anonymous reviewers, which helped us improve the paper. Xiangyi Ye was sponsored by the University of Michigan Undergraduate Research Opportunity Program (UROP). 


\section{References}

Somnath Banerjee, Krishnan Ramanathan, and Ajay Gupta. 2007. Clustering short texts using Wikipedia. In Proceedings of the 30th Annual International ACM SIGIR Conference on Research and Development in Information Retrieval, pages 787788. ACM.

Pavel Berkhin. 2006. A survey of clustering data mining techniques. In Grouping Multidimensional Data, pages 25-71. Springer.

Vincent D. Blondel, Jean-Loup Guillaume, Renaud Lambiotte, and Etienne Lefebvre. 2008. Fast unfolding of communities in large networks. Journal of statistical mechanics: theory and experiment, 2008(10):P10008.

Jianpeng Cheng and Dimitri Kartsaklis. 2015. Syntaxaware multi-sense word embeddings for deep compositional models of meaning. arXiv preprint arXiv:1508.02354.

William E. Donath and Alan J. Hoffman. 1973. Lower bounds for the partitioning of graphs. IBM Journal of Research and Development, 17(5):420-425.

Marcelo Luis Errecalde, Diego Alejandro Ingaramo, and Paolo Rosso. 2010. A new anttree-based algorithm for clustering short-text corpora. Journal of Computer Science \& Technology, 10.

Mark Everingham, Luc Van Gool, Christopher K. I. Williams, John Winn, and Andrew Zisserman. 2010. The PASCAL visual object classes (VOC) challenge. International Journal of Computer Vision, 88(2):303-338.

Brendan J. Frey and Delbert Dueck. 2007. Clustering by passing messages between data points. Science, 315(5814):972-976.

Hua He, Kevin Gimpel, and Jimmy Lin. 2015. Multiperspective sentence similarity modeling with convolutional neural networks. In Proceedings of the 2015 Conference on Empirical Methods in Natural Language Processing, pages 1576-1586.

Michael Heilman and Noah A. Smith. 2010. Tree edit models for recognizing textual entailments, paraphrases, and answers to questions. In Human Language Technologies: The 2010 Annual Conference of the North American Chapter of the Association for Computational Linguistics, HLT' 10, pages 1011-1019, Stroudsburg, PA, USA. Association for Computational Linguistics.

Sepp Hochreiter and Jürgen Schmidhuber. 1997. Long short-term memory. Neural Computation, 9(8):1735-1780.

Lawrence Hubert and Phipps Arabie. 1985. Comparing partitions. Journal of Classification, 2(1):193218.
Tom Kenter and Maarten de Rijke. 2015. Short text similarity with word embeddings. In Proceedings of the 24th ACM International on Conference on Information and Knowledge Management, pages 14111420. ACM.

Ben King, Rahul Jha, Dragomir R Radev, and Robert Mankoff. 2013. Random walk factoid annotation for collective discourse. In Proceedings of the 51st Annual Meeting of the Association for Computational Linguistics.

Ryan Kiros, Yukun Zhu, Ruslan R. Salakhutdinov, Richard Zemel, Raquel Urtasun, Antonio Torralba, and Sanja Fidler. 2015. Skip-thought vectors. In Advances in Neural Information Processing Systems, pages 3276-3284.

Matt Kusner, Yu Sun, Nicholas Kolkin, and Kilian Q. Weinberger. 2015. From word embeddings to document distances. In Proceedings of the 32nd International Conference on Machine Learning (ICML-15), pages 957-966.

Ding Liu and Daniel Gildea. 2005. Syntactic features for evaluation of machine translation. In Proceedings of the ACL Workshop on Intrinsic and Extrinsic Evaluation Measures for Machine Translation and/or Summarization, pages 25-32.

Chenglong Ma, Weiqun Xu, Peijia Li, and Yonghong Yan. 2015. Distributional representations of words for short text classification. In Proceedings of NAACL-HLT, pages 33-38.

Tomas Mikolov, Ilya Sutskever, Kai Chen, Greg S. Corrado, and Jeff Dean. 2013. Distributed representations of words and phrases and their compositionality. In Advances in Neural Information Processing Systems, pages 3111-3119.

Mark E. J. Newman. 2004. Analysis of weighted networks. Physical Review E, 70(5):056131.

Mark E. J. Newman. 2006. Modularity and community structure in networks. Proceedings of the $\mathrm{Na}$ tional Academy of Sciences, 103(23):8577-8582.

Mark Newman. 2010. Networks: An Introduction. Oxford University Press.

Andrew Y. Ng, Michael I. Jordan, and Yair Weiss. 2001. On spectral clustering: Analysis and an algorithm. In Advances in Neural Information Processing Systems, pages 849-856. MIT Press.

F. Pedregosa, G. Varoquaux, A. Gramfort, V. Michel, B. Thirion, O. Grisel, M. Blondel, P. Prettenhofer, R. Weiss, V. Dubourg, J. Vanderplas, A. Passos, D. Cournapeau, M. Brucher, M. Perrot, and E. Duchesnay. 2011. Scikit-learn: Machine learning in Python. Journal of Machine Learning Research, 12:2825-2830. 
Henry Petersen and Josiah Poon. 2011. Enhancing short text clustering with small external repositories. In Proceedings of the Ninth Australasian Data Mining Conference-Volume 121, pages 79-90. Australian Computer Society, Inc.

Vahed Qazvinian and Dragomir R. Radev. 2011. Learning from collective human behavior to introduce diversity in lexical choice. In Proceedings of the 49th Annual Meeting of the Association for Computational Linguistics: Human Language Technologies - Volume 1, HLT '11, pages 1098-1108, Stroudsburg, PA, USA. Association for Computational Linguistics.

Dragomir R. Radev, Amanda Stent, Joel R. Tetreault, Aasish Pappu, Aikaterini Iliakopoulou, Agustin Chanfreau, Paloma de Juan, Jordi Vallmitjana, Alejandro Jaimes, Rahul Jha, and Robert Mankoff. 2015. Humor in collective discourse: Unsupervised funniness detection in the new yorker cartoon caption contest. CoRR, abs/1506.08126.

Aniket Rangrej, Sayali Kulkarni, and Ashish V. Tendulkar. 2011. Comparative study of clustering techniques for short text documents. In Proceedings of the 20th International Conference Companion on World Wide Web, WWW'11, pages 111-112, New York, NY, USA. ACM.

Cyrus Rashtchian, Peter Young, Micah Hodosh, and Julia Hockenmaier. 2010. Collecting image annotations using amazon's mechanical turk. In Proceedings of the NAACL HLT 2010 Workshop on Creating Speech and Language Data with Amazon's Mechanical Turk, pages 139-147. Association for Computational Linguistics.

Kevin Dela Rosa, Rushin Shah, Bo Lin, Anatole Gershman, and Robert Frederking. 2011. Topical clustering of tweets. Proceedings of the ACM SIGIR: SWSM.

Frane Šarić, Goran Glavaš, Mladen Karan, Jan Šnajder, and Bojana Dalbelo Bašić. 2012. Takelab: Systems for measuring semantic text similarity. In Proceedings of the First Joint Conference on Lexical and Computational Semantics, pages 441-448. Association for Computational Linguistics.

Jianbo Shi and Jitendra Malik. 2000. Normalized cuts and image segmentation. Technical report.

Prajol Shrestha, Christine Jacquin, and Béatrice Daille, 2012. Computational Linguistics and Intelligent Text Processing: 13th International Conference, CICLing 2012, New Delhi, India, March 11-17, 2012, Proceedings, Part II, chapter Clustering Short Text and Its Evaluation, pages 169-180. Springer Berlin Heidelberg, Berlin, Heidelberg.

Richard Socher, Eric H. Huang, Jeffrey Pennin, Christopher D. Manning, and Andrew Y. Ng. 2011. Dynamic pooling and unfolding recursive autoencoders for paraphrase detection. In Advances in
Neural Information Processing Systems, pages 801809.

Kai Sheng Tai, Richard Socher, and Christopher D. Manning. 2015. Improved semantic representations from tree-structured long short-term memory networks. arXiv preprint arXiv:1503.00075.

Yun Tian, Haisheng Li, Qiang Cai, and Shouxiang Zhao. 2010. Measuring the similarity of short texts by word similarity and tree kernels. In Information Computing and Telecommunications (YC-ICT), 2010 IEEE Youth Conference on, pages 363-366. IEEE.

Stijn Van Dongen. 2000. Graph Clustering by Flow Simulation. Ph.D. thesis, University of Utrecht.

Jun Wang, Yiming Zhou, Lin $\mathrm{Li}$, Biyun $\mathrm{Hu}$, and Xia Hu. 2009a. Improving short text clustering performance with keyword expansion. In The Sixth International Symposium on Neural Networks (ISNN 2009), pages 291-298. Springer.

Kai Wang, Zhaoyan Ming, and Tat-Seng Chua. 2009b. A syntactic tree matching approach to finding similar questions in community-based qa services. In Proceedings of the 32nd International ACM SIGIR Conference on Research and Development in Information Retrieval, SIGIR '09, pages 187-194, New York, NY, USA. ACM.

Yu Wang, Lihui Wu, and Hongyu Shao. 2014. Clusters merging method for short texts clustering. Open Journal of Social Sciences, 2(09):186.

Peng Wang, Jiaming $\mathrm{Xu}$, Bo Xu, Cheng-Lin Liu, Heng Zhang, Fangyuan Wang, and Hongwei Hao. 2015. Semantic clustering and convolutional neural network for short text categorization. In Proceedings of the 53rd Annual Meeting of the Association for Computational Linguistics and the 7th International Joint Conference on Natural Language Processing, volume 2, pages 352-357.

Matthijs J. Warrens. 2008. On the equivalence of cohen's kappa and the hubert-arabie adjusted rand index. Journal of Classification, 25(2):177-183.

Jiaming $\mathrm{Xu}$, Peng Wang, Guanhua Tian, Bo Xu, Jun Zhao, Fangyuan Wang, and Hongwei Hao. 2015. Short text clustering via convolutional neural networks. In Proceedings of NAACL-HLT, pages $62-$ 69.

Xiaohui Yan, Jiafeng Guo, Shenghua Liu, Xue-qi Cheng, and Yanfeng Wang. 2012. Clustering short text using ncut-weighted non-negative matrix factorization. In Proceedings of the 21st ACM International Conference on Information and Knowledge Management, pages 2259-2262. ACM.

Jie Yin. 2013. Clustering microtext streams for event identification. In IJCNLP, pages 719-725. 
Min Zhang, Jian Su, Danmei Wang, Guodong Zhou, and Chew Lim Tan. 2005. Discovering relations between named entities from a large raw corpus using tree similarity-based clustering. In Natural Language Processing-IJCNLP 2005, pages 378-389. Springer. 